\title{
First-order Synchronization Transition in Locally Coupled Maps
}

\author{
P. K. Mohanty \\ Department of Physics of Complex Systems, Weizmann Institute of Science, Rehovot, Israel 76100.
}

(Dated: November 21, 2018)

\begin{abstract}
We study a family of diffusively coupled chaotic maps on periodic $d$-dimensional square lattices. Even and odd sub-lattices are updated alternately, introducing an effective delay. As the coupling strength is increased, the system undergoes a first order phase transition from a multi-stable to a synchronized phase. Further increase in coupling strength shows de-synchronization where the phase space splits into two ergodic regions. We argue that the de-synchronization transition is discontinuous for piece-wise linear maps, and is continuous for non-linear maps which are differentiable.
\end{abstract}

PACS numbers: 05.45.-a, 05.45.Xt

Synchronization is observed in a wide class of complex systems. Typically, it appears when the range of the correlations inside the system is of the same order as the system size. Few examples of mutual synchronization in complex dynamical systems are flashing fireflies 1], electronic circuits [2] and chemical reactions [3]. In recent years, synchronization of spatially extended systems has drawn considerable interest. In particular coupled map lattices (CMLs) [4], initially introduced as simple models of spatio-temporal chaos, has received a great deal of attention as a model of synchronization. It has been realized that two different replicas of the same CML are coupled directly [5] or through a common external random noise [6], can become synchronized for large enough coupling strengths. Recently, Ahlers and Pikovsky [] pointed out that synchronization transitions in one dimensional (1- $d$ ) CMLs are generically in the universality class of the Kardar-Parisi-Zhang (KPZ) model, however strongly nonlinear maps could be in the universality class of Directed percolation (DP).

From a point of view of general statistical theory synchronization is a non-equilibrium phase transition where distinct patches of the CMLs oscillate in phase. The phenomenon is similar to the roughening of growing interfaces and can be modeled by multiplicative noise partial differential equation 8 . This picture is further modified by Mũnoz and Pastor-Satorass $\underline{9}$ to incorporate first order phase transitions (FOPTs). However, it was not clear if the FOPTs observed in these models were just transients of DP. In context of non-equilibrium wetting process FOPTs are found in several $(1+1)$-dimensional stochastic models with local interactions [10, contrary to equilibrium wetting process where phase transitions are not possible [11] in 1-d systems having short-range interactions between interfaces and substrates. To the best of our knowledge FOPTs in deterministic, chaotic, extended systems with short range interactions are still lacking, although it is known to exist for globally coupled maps [12].

In this Letter we introduce and study a single parameter family of piece-wise linear chaotic maps which are diffusively coupled on a $d$-dimensional square lattice. A delay is introduced dynamically between sub-lattices by updating them alternately. One of our interests would be to find if, starting from a random initial condition, these sub-lattices synchronize at later times. The answer turns out to be 'no', for both very high and low diffusion strengths $\epsilon$. However for intermediate $\epsilon$ synchronization occurs with the suppression of spatio-temporal chaos. This synchronized phase is an unique absorbing state of the system and for piece-wise linear maps (PLMs) the phase boundary is identical with the boundary for a stable fixed point.

For $\epsilon=0$, the system visits the whole phase-space in time. The phase-space volume decreases as $\epsilon$ is increased and at a critical strength $\epsilon_{A}$, the phase-space suddenly shrinks to a point which corresponds to the fixed point of the primitive map. This fixed point is stable until $\epsilon=\epsilon_{B}>\epsilon_{A}$. Further increase in $\epsilon$ results in de-synchronization where the phase space splits into two ergodic regions about the collective bifurcation points. It may be argued that for PLMs the width of the bifurcation is independent of $\epsilon$ and thus synchronization error is discontinuous at the critical point. However for non-linear differentiable maps the width of the bifurcation vanishes as $\sqrt{\epsilon-\epsilon_{c}}$ which results in a continuous transition. We argue that this continuous transition is in a different universality class than that of KPZ and DP with critical exponents $\beta=1 / 2, \beta / \nu=0$ and $\gamma=1$ in 1 -dimension.

The Model : Consider a $d$-dimensional hyper-cubic lattice $\mathcal{L}$ of coupled identical maps $f\left(m, z_{\vec{i}}\right)$, where $z_{\vec{i}}$ is a real variable at site $\vec{i} \equiv\left(i_{1}, i_{2} \ldots i_{d}\right)$ with $i_{k}$ varying from 1 to $L$. We define the even and odd sub-lattices $\left(\mathcal{L}^{e}\right.$ and $\mathcal{L}^{o}$ respectively) as $\mathcal{L}^{e, o}=\left\{\vec{i}: \sum_{k} i_{k}=\right.$ even,odd $\}$, and denote $x_{\vec{i}}\left(y_{\vec{i}}\right)$ as the variable of $\mathcal{L}^{e}\left(\mathcal{L}^{o}\right)$. Starting from a random initial configuration, $\left\{x_{\vec{i}}\right\}$ and $\left\{y_{\vec{i}}\right\}$ are updated alternately as

$$
\begin{aligned}
& x_{\vec{i}}^{t+1}=(1-\epsilon) f\left(x_{\vec{i}}^{t}\right)+\frac{\epsilon}{2 d} \sum_{\vec{j} \in \mathcal{N}_{\vec{i}}} f\left(y_{\vec{j}}^{t}\right), \\
& y_{\vec{i}}^{t+1}=(1-\epsilon) f\left(y_{\vec{i}}^{t}\right)+\frac{\epsilon}{2 d} \sum_{\vec{j} \in \mathcal{N}_{\vec{i}}} f\left(x_{\vec{j}}^{t+1}\right),
\end{aligned}
$$

where $\mathcal{N}_{\vec{i}}$ is a set of $2 d$ nearest neighbors of $\vec{i}$ and $\epsilon$ is the coupling strength, can be seen as a diffusion constant. Equivalently, in the first half unit of time $\left\{x_{\vec{i}}\right\}$ are updated while $\left\{y_{\vec{i}}\right\}$ are at rest and the reverse happens in second half. We will see later that the delay which is in- 
troduced dynamically between sub-lattices is responsible for a complete synchronization of the system. Note that periodic boundary configuration in all $d$ dimensions are used throughout.

Synchronization occurs when the difference between $z_{\vec{i}}$ and its neighbors vanish at all sites as $t \rightarrow \infty$. Thus, the order parameter can be defined as $\phi=\left\langle\phi^{t}\right\rangle$ where

$$
\phi^{t}=\frac{1}{2 d L^{d}} \sum_{\vec{i} \in \mathcal{L}} \sum_{\vec{j} \in \mathcal{N}_{\vec{i}}}\left|z_{\vec{i}}^{t}-z_{\vec{j}}^{t}\right|,
$$

and steady state average is taken over time and realizations. Obviously $\phi$ vanishes in the synchronized phase and in the unsynchronized phase $\phi>0$. A trivial synchronized phase would correspond to the stable fixed point of the CLM, $i e,\left\{z_{\vec{i}}=z^{*}\right\}$. It is easy to see from Eq. (11) that $z^{*}=f\left(m, z^{*}\right)$. For chaotic CLMs without delay the largest Lyapunov exponent is independent of $\epsilon$ and is the same as the Lyapunov exponent of primitive map which is positive. Hence, a fixed point solution $\left\{z_{\vec{i}}=z^{*}\right\}$ is unstable for any $\epsilon$. With a delay, however, it can become negative in a region $\epsilon_{B} \leq \epsilon \leq \epsilon_{A}$. For PLMs $\epsilon_{A, B}$ corresponds to the boundary of linearly stable region which can be calculated as follows.

Let us take the initial state to be close to the fixedpoint, i.e., $x_{\vec{i}}=z^{*}+\delta x_{\vec{i}}$ and $y_{\vec{i}}=z^{*}+\delta y_{\vec{i}}$. In Fourierspace the evolution of $\delta x_{\vec{i}}$ and $\delta y_{\vec{i}}$ reads as,

$$
\left(\begin{array}{c}
\delta x_{\vec{k}} \\
\delta y_{\vec{k}}
\end{array}\right)^{t+1}=\left(\begin{array}{cc}
\tilde{\mu} & R_{\vec{k}} \\
\tilde{\mu} R_{\vec{k}} & \tilde{\mu}+R_{\vec{k}}^{2}
\end{array}\right)\left(\begin{array}{c}
\delta x_{\vec{k}} \\
\delta y_{\vec{k}}
\end{array}\right)^{t}
$$

$$
\text { where } R_{\vec{k}}=\frac{\epsilon \mu}{2 d} \sum_{\vec{r} \in \mathcal{N}_{\overrightarrow{0}}} e^{i \vec{k} . \vec{r}}, \mu=f^{\prime}\left(m, z^{*}\right)
$$

and $\tilde{\mu}=\mu(1-\epsilon)$. Let $E_{ \pm}$denote the eigenvalues of the matrix defined in Eq. (3). From the stability requirements $\left|\operatorname{Max}\left(\mathcal{R}\left(E_{+}\right), \mathcal{R}\left(E_{-}\right)\right)\right|<1$,

we find $\epsilon_{A}=1-1 / \mu$ and $\epsilon_{B}=(\mu+1) /(2 \mu)$ which are drawn in the inset of Fig. 1 1 as a phase boundary for the synchronized phase. For simplicity, it is assumed here that primitive maps have only single nonzero fixed point $z^{*}$. One can further generalize it to maps with more fixed points.

To find out the behavior of $\phi$ close to these transitions we first restrict ourselves to one dimension and study a specific single parameter family of maps :

$$
f(m, z)=\left\{\begin{array}{ll}
m z /(m-1) & z<a \\
m(1-z) & z \geq a
\end{array},\right.
$$

where $a=1-1 / m$. This piece-wise linear mapping of $[0,1]$ onto itself is everywhere expanding for $m>1$, and thus chaotic, with an invariant density uniform on $[0,1]$. A particular example of this family with $m=2$ is known as tent map. Note that the fixed point is $z^{*}=m /(m+1)$.

Synchronization : Let us first discuss the transition from the unsynchronized phase $A$ (inset of Fig. 1) to

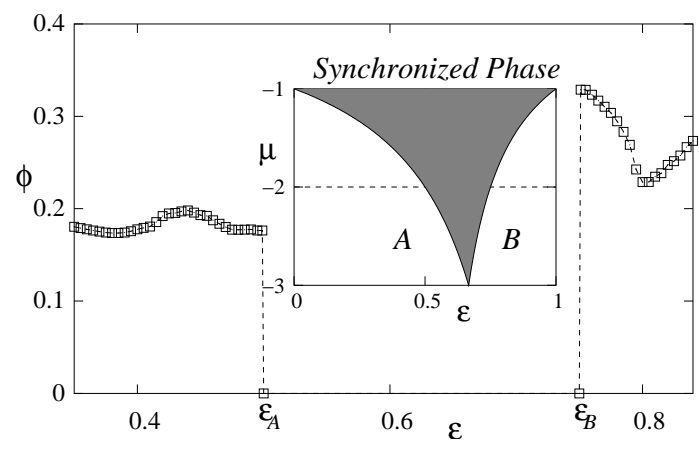

FIG. 1: Numerically obtained $\phi$ is plotted against $\epsilon$ for tent map $(\mu=-2)$. The inset shows the phase diagram in $\epsilon-\mu$ plane.

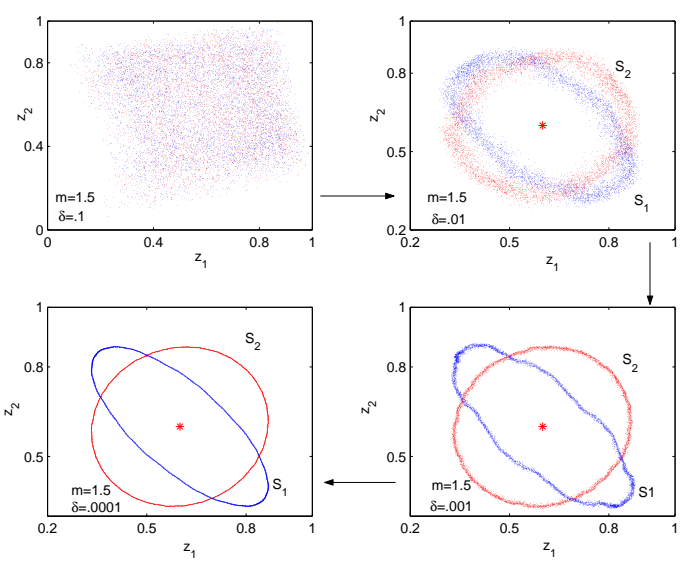

FIG. 2: This figure shows how the phase space changes in $z_{1}$ $z_{2}$ plane as $\epsilon \rightarrow \epsilon_{A}$; equivalently $\delta \rightarrow 0$. For large $\delta$, the phase space is identical for two different initial configurations $S_{1}$ and $S_{2}$. However, dynamically different shapes are generated as $\delta \rightarrow 0$. The symbol ' $*$ ' represents the fixed point $z^{*}=.6$.

the synchronized phase. Close to the transition we take $\epsilon=\epsilon_{A}-\delta$ and find that the system become multi-stable as $\delta \rightarrow 0$, i.e., there are large number steady states and one out of them is chosen by the system depending on the initial configuration. Multi-stability has been discussed earlier in the context of CMLs [13], and systems of delayed differential equations 14]. It may be argued that the multi-stability is extensive, i.e., the number of attractors grow exponentially with the system size. Thus any statistical average has to be taken over large number of independent realizations, which resticts us to simmulate large systems. We carried out numerical simmulations for $L=1024$ and $m=2$ (tent map) and find that $\phi$ vanishes discontinuously at $\epsilon_{A}=0.5$ (see Fig. 1). To confirm that it is a true first order transition, not just a transient effect, we monitor the phase space of every neighboring pair of co-ordinates as $\delta \rightarrow 0$. For example in Fig. 2] we demonstrate how the phase space changes in $z_{1}-z_{2}$ plane. Every other pair of neighboring co-ordinates show similar changes. In practice, no noticable change is observed in the phase space when $\delta<10^{-4}$ and then 
suddenly the fixed point $z_{1}=z^{*}=z_{2}$ appears at $\delta=0$. In other words, when $\delta \approx 0$, we have $\left|z_{i}-z_{i+1}\right|>0$ for every realization and thus $\phi$ has a jump at the critical point $\epsilon_{A}$.

De-synchronization : The synchronized state persists up to $\epsilon=\epsilon_{B}$ where phase space splits into two disconnected ergodic regions. In the new phase $B$ shown in the inset of Fig. 1 the system fluctuates about its collective bi-bifurcation points $x_{i}=x^{*}$ and $y_{i}=y^{*}$. Using Eq. (1) we get

$$
\begin{aligned}
& x^{*}=(1-\epsilon) f\left(x^{*}\right)+\epsilon f\left(y^{*}\right) \\
& y^{*}=(1-\epsilon) f\left(y^{*}\right)+\epsilon f\left(x^{*}\right),
\end{aligned}
$$

which can be solved for the family of maps defined in Eq. (4) as $x^{*}=\alpha_{ \pm}$and corresponding $y^{*}=\alpha_{\mp}$, with $\alpha_{ \pm}=\frac{m^{2}(2 \delta+1) \pm(2 \delta m+1)}{m^{2}(2 \delta+1)+m(4 \delta+1)}$ and $\delta=\epsilon-\epsilon_{B}$. Depending on the initial configuration, different parts of the sub-lattices are then attracted to $\alpha_{+}$or $\alpha_{-}$with kink-like interfaces (inset (a) of Fig. 3) separating them. It will be shown later that the width of such a kink $w$, diverges as $1 / \sqrt{\delta}$ as $\delta \rightarrow 0$. Thus, stable kinks can not be generated when $\delta$ is $\mathcal{O}\left(L^{-2}\right)$ and we have $\phi=\alpha_{+}-\alpha_{-}$. Clearly the jump in $\phi$ at the critical point $\epsilon_{B}$ is $\Delta=2 /\left(m^{2}+m\right)$. Note that in the other limit, i.e., when $L \rightarrow \infty$ before $\delta \rightarrow 0$, we have a slightly different $\phi$. In this case the large systems will generate an average density of kinks, say $\rho$. Immediately, we have $\phi=\Delta\left(1-\frac{\rho \mathcal{A}}{w \Delta}\right)$, where $\mathcal{A}$ is the area bounded by an even and an odd kink. Nevertheless the transition is discontinuous.

The profile of a kink can be calculated as follows. Let us assume that it starts at site $k$ with $z_{k}=\alpha_{-}$and $z_{k+1}=\alpha_{+}$. In steady state $z_{k+2}$ has two solutions : $\alpha_{-}$ and $\alpha=\frac{m^{2}(2 \delta+1)+(2 \delta m-1)}{m^{2}(2 \delta+1)+m(4 \delta+1)}$. It is obvious that the first solution, $z_{k+2}=\alpha_{-}$, does not generate a kink. Thus the steady state profile of a kink can be obtained as

$$
\left(\begin{array}{l}
z_{k+i+1} \\
z_{k+i+2}
\end{array}\right)=\left(\begin{array}{cc}
0 & 1 \\
-1 & -2 \cos (\theta)
\end{array}\right)^{i}\left(\begin{array}{c}
\alpha_{+} \\
\alpha
\end{array}\right)
$$

where $\theta=2 \tan ^{-1}\left(\sqrt{\delta / \epsilon_{B}}\right)$. Explicit form of $z_{k+i}$ is lengthy to present here, rather we compare numerically obtained $z_{k+i}$ with Eq. (6) in the inset (b) of Fig. 3 When site $i$ is far from $k$ and $\delta \approx 0$ one can write an approximate solution as $z_{k+2 i}=\alpha_{-}+\Delta_{i}$ and $z_{k+2 i+1}=\alpha_{-}-\Delta_{i}$, where $\Delta_{i}=\frac{i^{2} \delta}{2 m(m+1)}$. The width of the kink is thus $w=2 n$, such that $\Delta_{n}=\alpha_{+}-\alpha_{-}$. Clearly, $w$ diverges as $1 / \sqrt{\delta}$. In Fig 3 we have plotted $w$ obtained from numerical simulations versus $\delta$ in log-scale, which confirms this power-law.

Higher Dimensions : The phase diagram for coupled PLMs in higher dimension is the same as shown in the inset of Fig प Close to the transition point $\epsilon_{A}$, phase $A$ is mutistable and shows sudden shrink in phase space volume. The desynchronization transition occurs at $\epsilon_{B}$. But unlike one dimension, the kink-type solutions in phase $B$ are no more stable and hence jump in the orderparameter is $\Delta=2 /\left(m^{2}+m\right)$. Numerical simulations in 2 and 3 dimensions confirms this finding.

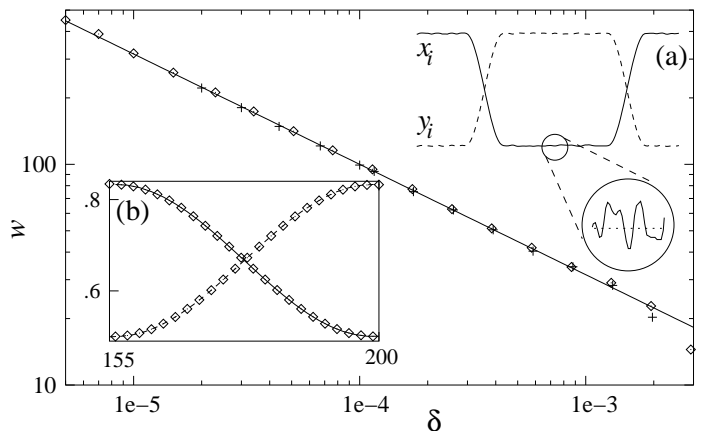

FIG. 3: A typical steady state profile of odd (solid-line) and even (dashed line) sub-lattices are shown in inset (a). Inset (b) compares the kink profile obtained from simulations (points) with Eq. (6). In the main figure, width of the kink $w$ is plotted against $\delta$ in log scale for $m=2$ and 2.5. The slope of the solid line is set to $-1 / 2$.

Other Maps : How do non-linear maps behave when a delay is introduced between sub-lattices ? Clearly the phase boundaries for synchronized phase strongly depends on corresponding primitive maps. First let us consider the logistic map : $f(x)=4 x(1-x)$. The fixed point of this map $z_{i}=z^{*}=3 / 4$ is linearly stable for $1 / 2<\epsilon<3 / 4$. However numerical simulation shows that the synchronization occurs at $\epsilon_{A}>1 / 2$. Close to the transition point phase $A$ is multistable and hence we have a first order transition similar to that of the tent map. The de-synchronization is found to occur exactly at $\epsilon_{B}$, where the linear-stability of the fixed point breaks down. Unlike the tent maps here we do not have fluctuations about the bifurcation points. But the kinks are unavoidable. Using Eqs. (5) and (6) one can obtain the bifurcation points as $x_{i}=\alpha_{ \pm}$and $y_{i}=\alpha_{\mp}$, where $\alpha_{ \pm}=\frac{8 \delta+3 \pm 2 \sqrt{\delta}(8 \delta+3)}{4(1+4 \delta)}$. Clearly $\Delta=\alpha_{+}-\alpha_{-}$vanishes as $\sqrt{\delta}$ indicating that the transition is continuous. Critical exponents of this continuous transition can be deduced using following arguments.

First, note that fluctuation of order-parameter comes about from the variation in density of kinks which fluctuates about a mean density, say $\rho$. Density distribution about $\rho$ can be assumed to be normal, which gives $\phi \sim \sqrt{\delta}\left(1-\frac{\mathcal{A} \rho}{w \Delta}\right)$, where $\mathcal{A}$ is the area bounded by an odd and an even kink, and $w$ is the width of the kink. Since $\phi$ is independent of $L$ and proportional to $\sqrt{\delta}$, we have critical exponents $\beta=1 / 2$ and $\beta / \nu=0$. One can further define "susceptibility" as $\chi=\left(\left\langle\phi^{2}\right\rangle-\langle\phi\rangle^{2}\right) / L$. Now $\chi \sim \delta r^{2} / L$, and hence $\gamma=1$. It is evident that the critical dimension $d_{c}=1$.

We have also done numerical simulations on sine $(f(x)=\sin (\pi x))$ and cubic $\left(f(x)=\sqrt{27} x\left(1-x^{2}\right) / 2\right)$ maps in one dimension, which show that the synchronization transition is discontinuous, whereas desynchronization occurs continuously with the same critical exponents as that of the logistic map.

To get an insight why de-synchronization is continuous 


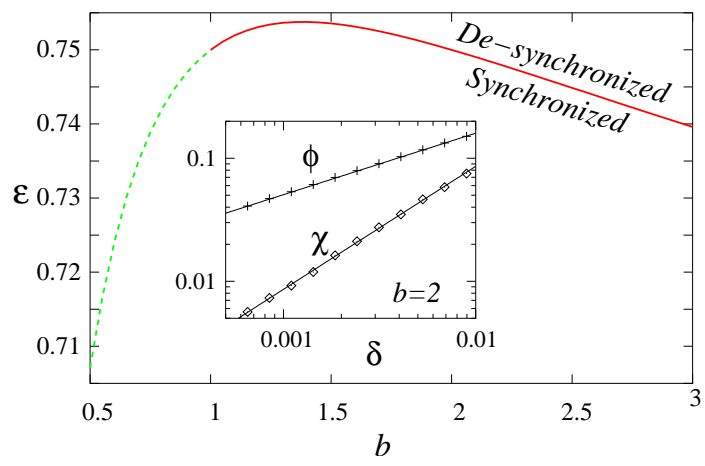

FIG. 4: Phase diagram of de-synchronization transition is shown in $\epsilon-b$ plane. The nature of transition changes from being discontinuous (dashed line) to continuous (solid line) at $b=1$. In the inset, $\phi$ and $\chi$ versus $\delta$ is plotted in log-scale and the solid lines are drawn with slope $\beta=1 / 2$ and $\gamma=1$ respectively for comparision.

for certain class of maps we investigate coupled powerlaw maps: $f(z)=1-|2 z-1|^{b}$, where $b=1$ and 2 corresponds to the tent and the logistic maps respectively. Thus, by tuning $b$ it is possible to study how the nature of transition changes from being first to second order. Our numerical simulations suggests that for $b>1$ the de-synchronization transition is continuous and belongs to the same universality class as that of the logistic map, whereas for $1 / 2<b \leq 1$ it is discontinuous. Note that there are two non zero fixed points for $b<1 / 2$ and present analysis need to be modified for their discussion . Basically, synchronization error is the average difference between bifurcation points $x^{*}$ and $y^{*}$, which vanishes at $\epsilon_{B}$ where $x^{*}=1 / 2=y^{*}$. Just above $\epsilon_{B}$ this difference is proportional to the difference between the left and right slope of $f(z)$ at $z=1 / 2$. For $b<1$, since these maps are not differentiable at $z=1 / 2$ the de-syncronization occurs discontinuously, whereas for $b>1$ the maps are differentiable and thus we have a continuous transition.

A few comments are in order. First, the synchronized phase discussed here is different from earlier studies, where CMLs remain chaotic in the synchronized phase for both, when they are coupled directly [5] or through noise [6]. In our model the spatio-temporal chaos are suppressed and thus the synchronized phase is a completely ordered phase. The spatial correlations even persist in de-synchronized phase $B$. Second, one can define a different order parameter called "magnetization" $M=\left\langle m^{t}\right\rangle$, where $m^{t}=\sum_{i} \operatorname{sign}\left(z_{i}^{t}-z^{*}\right)$. Then the desynchronyzation transition can be thought as a transition from fully ferromagnetic to fully antiferrmagnetic phase, which can be either first or second order. Note that the continuous transition obtained here is in a different universality class than that of zero temperature Ising transition in one dimension.

Conclusion : In conclusion, we study a single parameter family of coupled chaotic maps by introducing a delay between sub-lattices. We show that these systems undergo a first order transition to a synchronized phase when the coupling parameter is varied. The transition occurs as the system enters from a multiply stable region to a single 'collective fixed point' in phase space. A second transition occurs as this fixed point become unstable and the phase space breaks up into two ergodic regions about the collective bifurcation points. Our analytical results show that the de-synchronization transition is discontinuous in one and higher dimensions when the primitive maps are not differentiable. Differentiable maps also show a first-order synchronization transition, whereas the de-synchronization occurs continuously with critical exponents $\beta=1 / 2, \gamma=1$, and $\nu^{-1}=0$. We argue that the de-synchronization transition discussed here can also be thought as a transition from a fully ferromagnetic to a fully anti-ferromagnetic phase. These results establish a true non-equilibrium first order transition in one dimensional dynamical systems with short range interactions.

Acknowledgments : We thank D. Mukamel and E. Levin for fruitful comments and discussions.
[1] J. Buck, Quart. Rev. Biol. 63, 265(1988).

[2] J. F. Heagy, T. L. Carroll, and L. M. Pecora, Phys. Rev. E 50, 1874 (1994).

[3] Y. Kuramoto, Chemical oscilations, Waves and Turbulence, Springer, Berlin (1984).

[4] Theory and Application of Coupled map Lattices, ed. by K. Kaneko, J. Wiley and Sons, Chichester, (1993).

[5] J. F. Heagy, T. L. Carroll, and L. M. Pecora, Phys. Rev. E 50, 1874(1994); P. Grassberger, Phys. Rev. E 59, R2520.

[6] F. Cecconi, R. Livi and A. Politi, Phys. Rev. E 57, 2703 (1998). L. Baroni, R. Livi, and A. Torcini, Phys. Rev. E 63, 036226 (2001).

[7] V. Ahlers, and A. Pikovsky, Phys. Rev. Lett. 88, 254101 (2002).

[8] A. S. Pikovsky and J. Kurths, Phys. Rev. E 49, 898 (1994).
[9] M. Mũnoj and R. Pastor-Satorras, Phys. Rev. Lett. 91, 190403 (2003).

[10] H. Hinrichsen, R. Livi, D. Mukamel and A. Politi, Phys. Rev. E 61, R1032 (2000); L. Giada, and M. Marsili, Phys. Rev. E 62, 6015 (2000).

[11] R. K. P. Zia, R. Lipowsky, and D. M. Kroll, Am. J. Phys. 56, 160 1(1998).

[12] R. Müller, K. Lippert, A. Kühnel, and U. Behn, Phys. Rev. E 56, 2658 (1997); S. Kim, S. H. Park and C. S. Ryn Phys. Rev. Lett. , 78, 1616 (1997).

[13] A. Lemaitre, and H. Chate', Phys. Rev. Lett. 82, 1140 (1999) and references there in.

[14] K. Ikeda, and K. Kondo, Phys. Rev. Lett. 49, 1467 (1982); J. Foss, A. Longtin, B. Mensour, and J. Milton, ibid 76, 708 (1996). 